Nervenarzt 2018 $89: 1043-1048$

https://doi.org/10.1007/s00115-017-0462-4

Online publiziert: 5. Dezember 2017

(c) Der/die Autor(en) 2017. Dieser Artikel ist eine Open-Access-Publikation.

CrossMark

\section{Hintergrund}

Die Bindungstheorie geht auf die Arbeiten von John Bowlby und Mary Ainsworth [3] zurück, wobei unter dem Bindungssystem ein biologisch und evolutionär verankertes Motivations- und Verhaltenssystem verstanden werden kann, welches

... über die Interaktion mit den Bindungsfiguren (meist den Eltern) vermittelt wird und sich seinerseits auf Affektregulation, Beziehungsgestaltung und deren neurobiologischer Korrelate auswirkt [23, S. 45].

Demnach wird das Bindungssystem durch das frühe familiäre Umfeld geprägt und im Laufe unseres Lebens immer wieder in unterschiedlichsten Situationen aktiviert $[3,15]$. Eine sichere Bindung schafft eine „sichere Basis“ für die Erkundung der Außenwelt sowie einen „sicheren Hafen“, in den bei Angst oder Stress zurückgekehrt werden kann. Steht diese Sicherheit nur eingeschränkt zur Verfügung, kann dies die Entstehung psychischer Erkrankungen begünstigen [2, 25]. So kann Bindung als Einflussgröße im Rahmen eines multidimensionalen Modells der Suchtentwicklung beschrieben werden, in welchem neurobiologische, soziale sowie psychologische Faktoren berücksichtigt werden [23].

\section{Bindung und Sucht}

Neben den vorherrschenden lerntheoretischen und klassisch orientierten psychoanalytischen Konzepten, bietet die Bindungstheorie einen weiteren möglichen Zugang zur Erklärung und Behandlung von Suchterkrankungen [9]. So erschweren defizitäre bzw. negative

\author{
H. F. Unterrainer ${ }^{1,2} \cdot$ M. Hiebler-Ragger ${ }^{1,2} \cdot$ L. Rogen ${ }^{1,2} \cdot$ H. P. Kapfhammer ${ }^{2}$ \\ 'Verein Grüner Kreis, Center for Integrative Addiction Research (CIAR), Wien, Österreich \\ ${ }^{2}$ Universitätsklinik für Psychiatrie und Psychotherapeutische Medizin, Medizinische Universität Graz, Graz, \\ Österreich
}

\title{
Sucht als Bindungsstörung
}

Beziehungserfahrungen eines Kindes das Erlernen adäquater Regulationsmechanismen für negative Affekte, wie beispielsweise Angst oder Frustration. Des Weiteren führt die Internalisierung dieser Bindungserfahrungen $\mathrm{zu}$ negativen „inneren Arbeitsmodellen“ (Beziehungserwartungen) bezüglich des eigenen Selbst und/oder anderen Personen. Die Drogeneinnahme kann in diesem Sinne als ein dysfunktionaler Versuch verstanden werden, Defizite in der Bindungsorganisation zu kompensieren $[24,32]$. Dem entsprechend

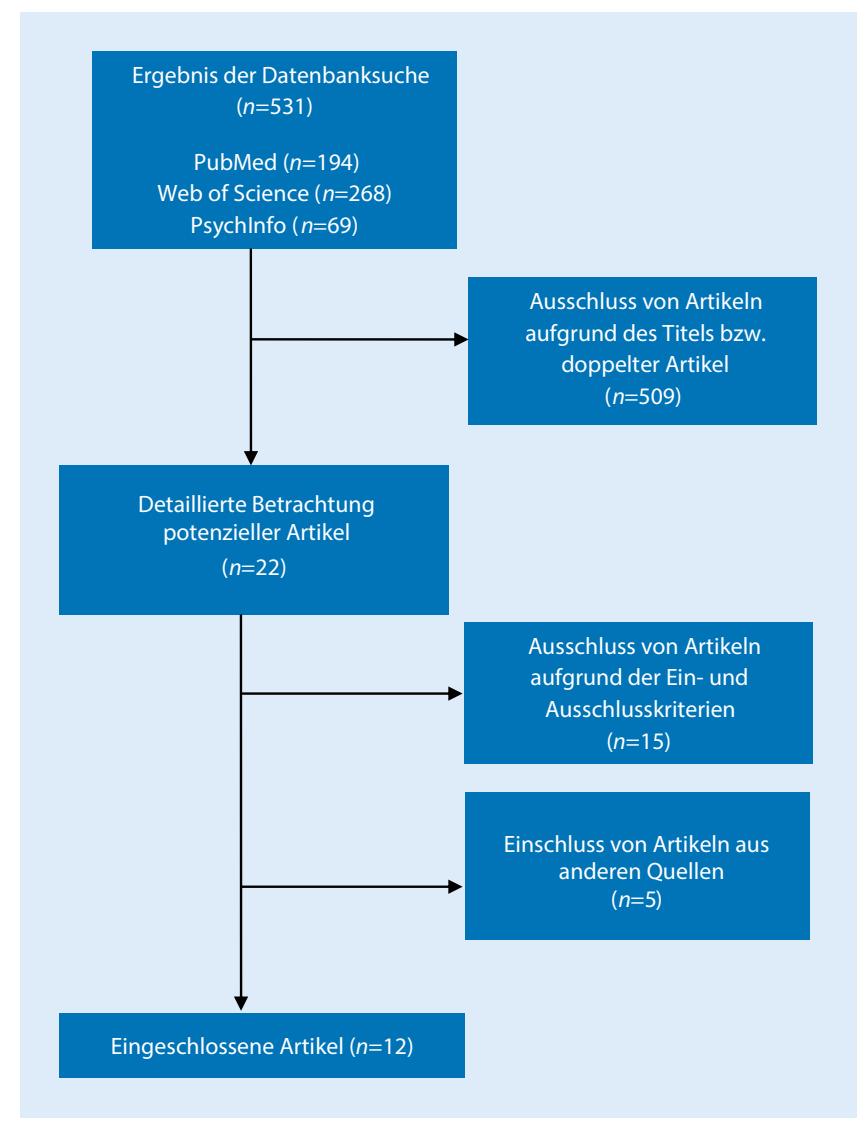

kann man bei Sucht auch als Form des exzessiven Appetits sprechen oder als

... Bindung an eine appetitive (lustgesteuerte) Aktivität, welche so stark ausgeprägt ist, dass es für die Person schwierig ist, diese Aktivität zu zügeln, obwohl diese einen Schaden verursacht [16, S. 18; Übersetzung: HFU].

\section{Messung des Bindungsverhaltens}

Basierend auf teilweise unterschiedlichen Konzeptualisierungen des Bindungskonstrukts, finden sich in der
Abb. 1 - Flowchart zum Ablauf der Literaturrecherche 
Literatur sehr heterogene Zugänge zur psychometrischen Erfassung von Bindungsmerkmalen. Dementsprechend weisen Kirchmann und Strauß [12] auf die besondere Problematik der mangelnden Konvergenz der verwendeten Methoden hin. Während es einige gut validierte (Selbstbeurteilungs-)Fragebögen zu Bindungsmerkmalen gibt $[4,26]$, wird diese Art der Operationalisierung auch häufig hinterfragt [10]. Da Unterschiede zwischen bewussten bzw. unbewussten Repräsentationen bzw. Selbst- und Fremdbild der jeweiligen Versuchsperson $\mathrm{zu}$ unterschiedlichen Ergebnissen führen könnten [5], ergibt sich die Notwendigkeit der Differenzierung von Ergebnissen, welche mithilfe von Fragebögen oder mittels halbstrukturierter Interviews zustande gekommen sind. Des Weiteren finden sich in der Literatur, ausgehend von dem Grundkonzept eines sicheren vs. unsicheren Bindungssytems, drei („sicher“, „ängstlich-ambivalent", „ängstlich-vermeidend“) bzw. auch vier („sicher“, „anklammernd“, „abweisend“, „ängstlich-vermeidend“) verschiedene Kategorien von Bindungstypen (vgl. [14] für eine ausführliche Übersicht).

In dieser Arbeit soll, mittels eines systematischen Überblicks empirischer Studien, der Fragestellung nachgegangen werden, inwieweit Sucht als mögliche Form der Bindungsstörung gelten darf. Die vorliegende Zusammenschau bisheriger Studien legt den Fokus auf substanzgebundene Süchte, da nur diese durch die etablierten Diagnosesysteme (mit Ausnahme des pathologischen Glücksspiels) ausreichend gut charakterisiert werden können. Um allgemeine Grundlagen zu schaffen, beschränken wir uns in dieser Arbeit zum großen Teil auf die globale Differenzierung zwischen sicherer und unsicherer Bindung und deren Zusammenhang mit substanzbezogenen Süchten. Darauf aufbauend können Nachfolgearbeiten auf spezielle unsichere Bindungsmuster im Zusammenhang mit einer bestimmten Substanzwahl eingehen.

Nervenarzt 2018 $\cdot 89: 1043-1048$ https://doi.org/10.1007/s00115-017-0462-4

๑ Der/die Autor(en) 2017. Dieser Artikel ist eine Open-Access-Publikation.

\section{H. F. Unterrainer $\cdot$ M. Hiebler-Ragger $\cdot$ L. Rogen · H. P. Kapfhammer}

\section{Sucht als Bindungsstörung}

\section{Zusammenfassung}

Hintergrund. Wenngleich es bis heute kein allgemein akzeptiertes Modell zur Beschreibung der Entstehung und Behandlung von Suchterkrankungen gibt, stellt die Bindungstheorie bereits seit Jahrzehnten eine bedeutsame Basis für den klinisch-therapeutischen Umgang mit Suchterkrankungen dar. In dieser Arbeit soll ein systematischer Überblick der empirischen Studien zum Zusammenhang zwischen substanzgebundenen Süchten und Störungen des Bindungsverhaltens gegeben werden.

Fragestellung. Gibt es einen positiven Zusammenhang zwischen der Störung des Bindungsverhaltens und dem Vorliegen einer Substanzabhängigkeit?

Methode. Zur Erfassung der relevanten Studien wurden verschiedene elektronische Datenbanken (Psychlnfo, Web of Science, PubMed) systematisch nach Journal- und Buchbeiträgen in englischer und deutscher Sprache durchsucht. Aus den Ergebnislisten wurden 22 Publikationen ausgewählt.
Nach der strengeren Beschränkung auf Originalarbeiten konnten letztlich 12 Artikel als passend akzeptiert werden.

Ergebnisse. In 10 von 12 empirischen Arbeiten zeigte sich ein relevanter Zusammenhang zwischen substanzgebundenem Suchtverhalten und unsicherer Bindung. Schlussfolgerungen. Durch die Ergebnisse bisheriger Forschungsarbeiten erhärtet sich die Hypothese, süchtiges Verhalten als möglichen Ausdruck einer Bindungsstörung zu verstehen. Damit wird auch die Bedeutsamkeit der Berücksichtigung bindungsbezogener Parameter in Prävention und Behandlung von substanzgebundenen Süchten unterstrichen. Weiterführende Arbeiten könnten auf nichtsubstanzgebundene Süchte bzw. Therapieverlaufsstudien fokussieren.

\section{Schlüsselwörter}

Bindungsstörung · Systematischer Überblick . Substanzmissbrauch - Messmethoden . Suchttherapie

\section{Addiction as an attachment disorder}

\section{Abstract}

Background. There is no commonly accepted model for describing the development and treatment of substance use disorders (SUD); however, over several decades the attachment theory has represented an important basis for the clinical handling of SUD. This study gives a systematic review of empirical studies regarding the relationship between SUD and disorders of attachment behavior.

Objective. Is there a positive relationship between disorders of attachment behavior and the presence of SUD?

Method. Various databases (Psychlnfo, Web of Science, PubMed) were systematically searched in order to pinpoint relevant studies in books and articles published in English or German. Based on the results 22 publications were selected. After a stricter limitation to original research, 12 articles could finally be accepted as eligible.

Results. A significant relationship was found between SUD and insecure attachment in 10 out of the 12 studies.

Conclusion. Based on the results of the research studies reviewed the hypothesis to characterize SUD as a possible expression of an attachment disorder was confirmed. This corroborates the importance of considering attachment parameters in dealing with prevention and treatment of SUD. Further research might focus on non-substancerelated addictive disorders and therapy outcome studies.

\section{Keywords}

Attachment disorder - Substance abuse . Systematic review - Measurement methods . Addiction treatment

\section{Methode}

Im Allgemeinen wurde bei der Erstellung des Überblicks auf die Einhaltung einschlägiger Richtlinien geachtet [27].
Wir haben uns dabei auf Arbeiten beschränkt, welche nach 1990 erschienen sind, um einen aktuellen Überblick zu gewährleisten. Hinsichtlich der Auswahl der Suchtbegriffe wurde ein ähnliches 


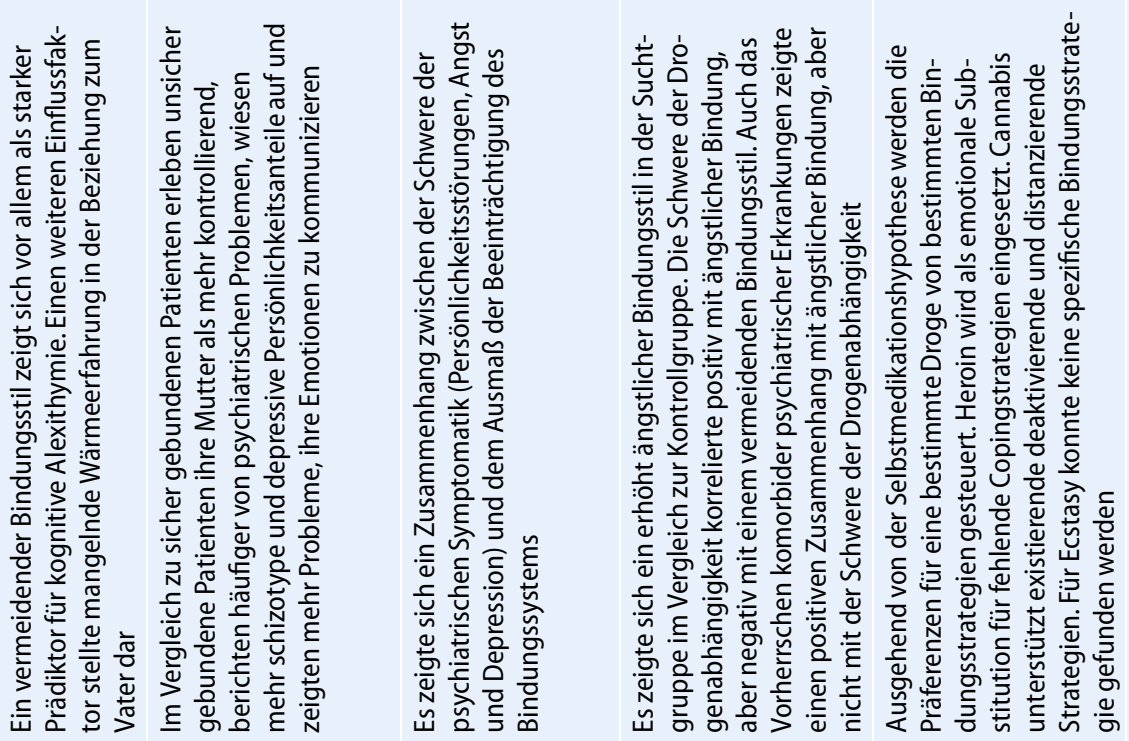

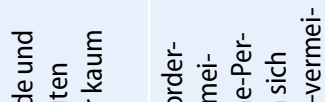

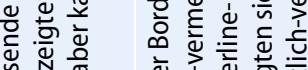

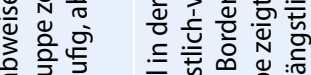

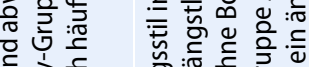

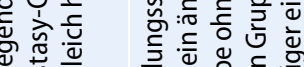
㟧苟

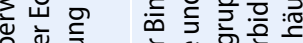

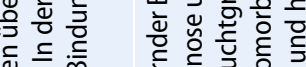

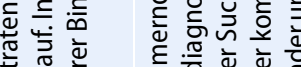

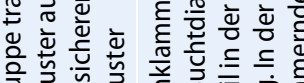

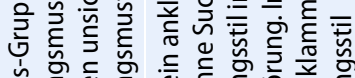

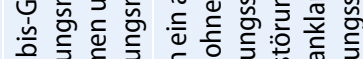

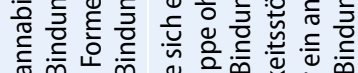
त् के के के 옹 के के के

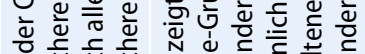

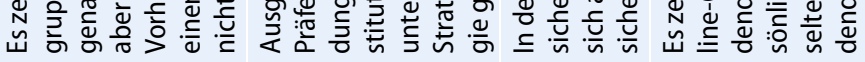

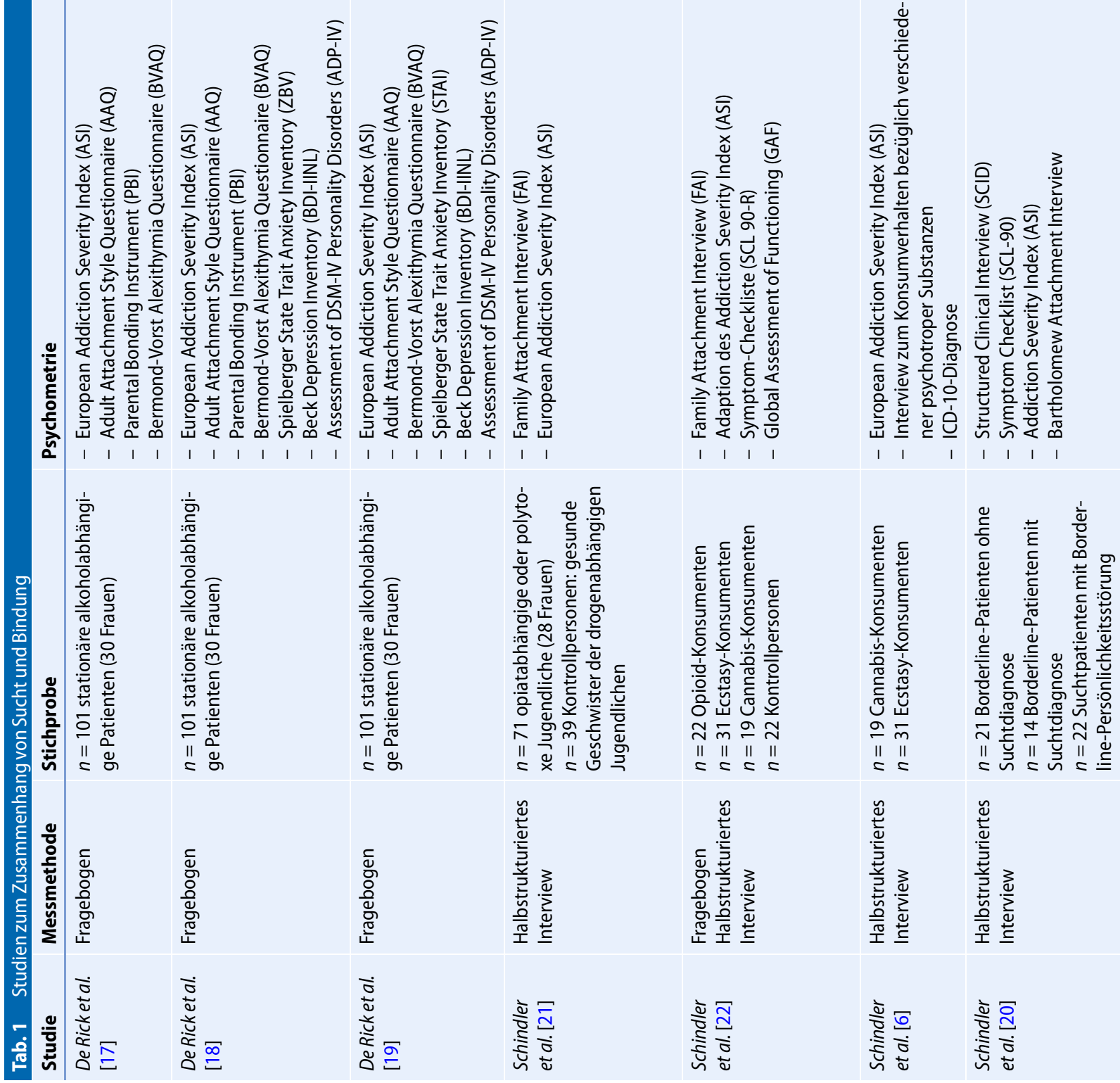


Vorgehen wie in früheren Übersichtsarbeiten gewählt. Vergleichbare Arbeiten finden sich von Schindler et al. [21] bzw. von Mikulincer und Shaver [14].

Zur Erfassung der bisher durchgeführten Studien zum Zusammenhang von substanzgebundener Sucht und Bindung wurden gängige elektronische $\mathrm{Da}$ tenbanken (PsychInfo, Web of Science, PubMed) systematisch durchsucht. Es wurde nach empirischen Studien, publiziert in Journal- und Buchbeiträgen, im Zeitraum von 1990 bis April 2017 in englischer und deutscher Sprache in den gängigen Datenbanken gesucht. Dabei wurden folgende Schlagwörter verwendet: „addiction“, „substance use disorder", „attachment", „attachment style“, „attachment disorder“, „insecure attachment" bzw. „Sucht“, „Substanzgebrauchsstörung“", „Bindung“", „Bindungsstile“, „Bindungsstörung“, „unsichere Bindung". Mit den angeführten Schlagwörtern konnten insgesamt 531 Einträge identifiziert werden.

Nach dem Ausschluss mehrfach gefundener bzw. nicht themenbezogener Arbeiten, wurden 22 Publikationen beibehalten. Bei 15 dieser Artikel handelte es sich um rein theoretische Diskussionen bzw. Übersichtsarbeiten. Diese wurden entfernt. Des Weiteren konnten 5 Arbeiten aufgrund einer erweiterten Recherche (Google Scholar) bzw. auch persönliche Empfehlungen miteinbezogen werden. Somit wurden letztlich 12 Artikel für die Analyse berücksichtigt (• Abb. 1).

\section{Ergebnisse}

Wie in $\bullet$ Tab. 1 veranschaulicht, konnten insgesamt 12 Studien für eine genaue inhaltliche Analyse herangezogen werden. Diese werden differenziert nach der Erhebungsmethode des Bindungsstils, Stichprobe bzw. Substanzwahl (,drug of choice"), dem Einsatz anderer psychometrischer Verfahren und der Darstellung der wichtigsten Ergebnisse hinsichtlich der Bindungsstile inhaltlich näher erläutert.

Aufgrund der drei Arbeiten von De Rick et al. [17-19] kann darauf geschlossen werden, dass eine verminderte Bindungssicherheit mit einem erhöhten Ausmaß an Psychopathologie einhergeht. 
In Korrespondenz dazu zeigt sich in der Arbeit von Schindler et al. [21] ein ängstlicher Bindungsstil mit der Schwere der Drogenabhängigkeit und mit komorbid auftretenden psychiatrischen Erkrankungen als positiv korreliert. Im Allgemeinen zeigte sich ein ängstlicher Bindungsstil innerhalb der Suchtgruppe im Vergleich zur Kontrollgruppe als deutlich erhöht. In Ergänzung dazu präsentieren Schindler et al. [22] Ergebnisse, welche darauf hinweisen, dass ein bestimmtes unsicheres Bindungsmuster für eine bestimmte Substanzwahl („drug of choice") prädisponieren könnte. Auch zeigten sich hier unsichere Bindungsstile in den Suchtgruppen als deutlich erhöht. Des Weiteren konnten Schindler et al. [20] einen ängstlich-vermeidenden Bindungsstil in einer Gruppe von Suchtpatienten mit bzw. ohne BorderlineDiagnose wiederfinden.

In den drei durchgesehenen Arbeiten von Hiebler-Ragger et al. [11] bzw. Unterrainer et al. [28, 29] konnte jeweils ein unsicherer Bindungsstil innerhalb der Suchtgruppen im Vergleich zu Kontrollgruppe beobachtet werden.

\section{Diskussion}

In der vorliegenden Arbeit sollte anhand der Zusammenstellung empirischer Arbeiten untersucht werden, ob sich eine Suchterkrankung als Form der Bindungsstörung charakterisieren lässt. Trotz der unsicheren Datenlage kann die Annahme eines gehäuften Auftretens unsicherer Bindung bei Suchtpatienten zumindest als tendenziell bestätigt gelten. Auch mag das Auftreten eines ängstlich-vermeidenden Bindungsstils als spezifisch für die Suchterkrankungen gelten [11, 17, 20, 21, 28, 29]. Dem entsprechend konnte z. B. auch das gehäufte Auftreten eines ängstlich-vermeidenden Bindungsstils bei Suchtpatienten im Vergleich zu Borderline-Patienten herausgearbeitet werden [20]. Halbstrukturierte Interviews fokussieren dabei eher auf eine kategoriale Einordnung nach verschiedenen Bindungsstilen, während Fragebögen meist auf eine dimensionale $\mathrm{Ab}$ bildung der Bindungssicherheit ausgelegt sind. Hier bleibt somit die Frage offen, inwieweit verschiedene Erhebungsmetho- den die Ergebnisse der Studien beeinflussen. Insgesamt wurden die durchgesehenen Arbeiten meist an kleinen Stichproben durchgeführt bzw. gab es teilweise keine Kontrollgruppe - das vermindert die Aussagekraft der Ergebnisse.

Die gesunden Vergleichsgruppen wiesen in allen Studien einen überwiegend sicheren Bindungsstil auf. Des Weiteren konnte in einzelnen Arbeiten ein Zusammenhang zwischen einem unsicheren Bindungsmuster und dem erhöhten Auftreten weiterer psychiatrischer Symptome (u.a. Depressionen und Persönlichkeitsstörungen) festgestellt werden [17, 18, 23]. Schindler et al. [20] weisen anhand unterschiedlicher Bindungsmuster bei Suchtpatienten darauf hin, dass hier möglicherweise der Versuch unternommen wird, durch unterschiedliche Substanzen spezifische Bindungsdefizite zu kompensieren. Demgegenüber konnten allerdings in einer anderen Studie keine Unterschiede zwischen Alkoholpatienten und polytoxikomanen Patienten hinsichtlich der Bindungsstile gefunden werden [11]. Trotzdem erscheint es für zukünftige Arbeiten sinnvoll, unterschiedliche Muster unsicheren Bindungsverhaltens im Zusammenhang mit Suchterkrankungen genauer zu betrachten.

Wir haben uns in der hier vorliegenden Arbeit auf die Darstellung der substanzgebundenen Süchte beschränkt, da nur für diese (mit Ausnahme des Glücksspiels) bereits eine allgemein akzeptierte Beschreibung in den gängigen Klassifikationssystemen (International Statistical Classification of Diseases and Related Health Problems [ICD] 10, Diagnostic and Statistical Manual of Mental Disorders [DSM] 5) vorliegt. Zukünftig erscheint die Berücksichtigung von Bindungsstilen für den Umgang mit substanzungebundenen Süchten, wie z. B. der Internetsucht, als durchaus vielversprechend zur verbesserten Charakterisierung substanzungebundener Suchterkrankungen bzw. auch zur besseren Abgrenzung von substanzgebundenen Süchten [7]. Im Allgemeinen unterstreichen die Ergebnisse die Bedeutsamkeit bindungsbasierter therapeutischer Interventionstechniken in der Suchttherapie [8]. Dementsprechend konnte in einer
Überblicksarbeit auch die Wichtigkeit der therapeutischen Beziehung am Beginn einer Suchtherapie für den weiteren Verlauf einer Behandlung herausgearbeitet werden [13], wobei einschränkend darauf verwiesen werden muss, dass dies nicht notwendigerweise die Bedeutung bindungsbasierter Interventionen unterstreicht [1].

\section{Fazit für die Praxis}

\section{- Der postulierte positive Zusammen- hang zwischen unsicherer Bindung und Suchterkrankung konnte anhand der Zusammenschau empirischer Ar- beiten tendenziell bestätigt werden. \\ - Unterschiedliche Messmethoden (Fragenbögen vs. halbstrukturierte Interviews) beeinträchtigen die Vergleichbarkeit der Studien. \\ - Teilweise ergeben sich Hinweise auf Zusammenhänge zwischen spezifischen Bindungsmustern und Substanzwahl. \\ - Als nächstes wichtiges Ziel ist die Erforschung der Veränderung von Bindungsmustern im Verlauf einer Suchttherapie zu nennen.}

\section{Korrespondenzadresse}

PD DDr. H. F. Unterrainer

Verein Grüner Kreis, Center for Integrative Addiction Research (CIAR)

Rudolfsplatz 9, 1010 Wien, Österreich

human.unterrainer@gruenerkreis.at

Funding. Open access funding provided by Medical University of Graz.

\section{Einhaltung ethischer Richtlinien}

Interessenkonflikt. H.F. Unterrainer, M. HieblerRagger, L. Rogen und H.P. Kapfhammer geben an, dass kein Interessenkonflikt besteht.

Dieser Beitrag beinhaltet keine von den Autoren durchgeführten Studien an Menschen oder Tieren.

Open Access. Dieser Artikel wird unter der Creative Commons Namensnennung 4.0 International Lizenz (http://creativecommons.org/licenses/by/4.0/deed. de) veröffentlicht, welche die Nutzung, Vervielfältigung, Bearbeitung, Verbreitung und Wiedergabe in jeglichem Medium und Format erlaubt, sofern Sie den/die ursprünglichen Autor(en) und die Quelle ordnungsgemäßnennen, einen LinkzurCreative Commons Lizenz beifügen und angeben, ob Änderungen vorgenommen wurden. 


\section{Literatur}

1. Atanassov N, Savov S (2013) Deficits of affect mentalization in patients with drug addiction: theoretical and clinical aspects. ISRN Addict. https://doi.org/10.1155/2013/250751

2. BelskyJ(1997) Attachment, mating, and parenting. Hum Nat 8:361-381

3. Bowlby J (2005) A secure base: clinical applications of attachment theory. Taylor \& Francis, Oxford

4. Collins NL, Read SJ (1990) Adult attachment, working models, and relationship quality in dating couples.J Jers Soc Psychol 58:644-663

5. Crowell JA, Treboux D (1995) A review of adult attachment measures: Implications for theory and research. SocDev 4:294-327

6. Rumpf H-J, Kiefer F, Deutsche Gesellschaft für Suchtforschung und Suchttherapie (2011) DSM5: Die Aufhebung der Unterscheidung von Abhängigkeit und Missbrauch und die Öffnung für Verhaltenssüchte: DSM-5: Removal of the Distinction between Dependence and Abuse and the Opening for Behavioural Addictions. Sucht 57:45-48

7. Eichenberg C, Dyba J, SchottM (2017) Bindungsstile, Nutzungsmotive und Internetsucht. Psychiatr Prax 44:41-46

8. Flores PJ (2001) Addiction as an attachment disorder: Implications for group therapy. Int J Group Psychoth 51:63-81

9. Flores PJ (2004) Addiction as an attachment disorder. Jason Aronson, Lanham

10. Grossmann KE, Grossmann K (2002) Einflüsse von Bindungspersonen auf die Entwicklung von Gefühlen, Motiven und Perspektiven über den Lebenslauf. In: Kretz H (Hrsg) Lebendige Psychohygiene 2000plus. Eberhard, München, S229-252

11. Hiebler-Ragger M, Unterrainer H-F, Rinner A et al (2016) Insecure attachment styles and increased borderline personality organization in substance use disorders. Psychopathology 49:341-344

12. Kirchmann H, Strauß B (2008) Methoden zur Erhebung von Bindungsmerkmalen. Klin Diagn Eval 1:293-327

13. Meier PS, Barrowclough C, Donmall MC (2005) The role of the therapeutic alliance in the treatment of substance misuse: a critical review of the literature. Addiction 100:304-316

14. Mikulincer M, Shaver PR (2007) Attachment in adulthood: structure, dynamics, and change. Guilford, New York

15. Milch W, Sahhar N (2010) Zur Bedeutung der Bindungstheorie für die Psychotherapie Erwachsener. Psychotherapie 15:44-55

16. Orford J (2001) Addiction as excessive appetite. Addiction 96:15-31

17. De Rick A, Vanheule S (2006) The relationship between perceived parenting, adult attachment style and alexithymia in alcoholic inpatients. Addict Behav 31:1265-1270

18. De Rick A, Vanheule S (2007) Attachment styles in alcoholic inpatients. Eur Addict Res 13:101-108

19. De Rick A, Vanheule S, Verhaeghe P (2009) Alcohol addiction and the attachment system: an empirical study of attachment style, alexithymia, and psychiatric disorders in alcoholic inpatients. Subst Use Misuse 44:99-114

20. Schindler A, Bröning S (2015) A review on attachment and adolescent substance abuse: empirical evidenceandimplicationsfor prevention and treatment. Subst Abuse 36:304-313

21. Schindler A, Thomasius R, Sack P-M et al (2005) Attachment and substance use disorders: a review of the literature and a study in drug dependent adolescents. Attach Hum Dev 7:207-228

22. Schindler A, Thomasius R, Petersen K et al (2009) Heroin as an attachment substitute? Differences in attachment representations between opioid, ecstasy and cannabis abusers. Attach Hum Dev 11:307-330

23. Schindler A, Sack P-M, Thomasius R (2012) Bindungsmuster von Cannabis-und Ecstasykonsumenten. Sucht 58:45-53

24. Schuengel C, Van ljzendoorn MH (2001) Attachment in mental health institutions: a critical review of assumptions, clinical implications, and research strategies. Attach Hum Dev 3:304-323

25. Sheinbaum T, Kwapil TR, Ballespí $S$ et al (2015) Attachment style predicts affect, cognitive appraisals, and social functioning in daily life. Front Psychol 6:296

26. Sibley CG, Fischer R, Liu JH (2005) Reliability and validity of the revised experiences in close relationships (ECR-R) self-report measure of adult romantic attachment. Pers Soc Psychol Bull 31:1524-1536

27. Moher D, Liberati A, Tetzlaff J, Altman DG, The PRISMA Group (2009) Preferred reporting items for systematic reviews and Meta-analyses: thePRISMA statement.PLoS Med 6(7):e1000097

28. Unterrainer $\mathrm{H}$, Hiebler M, Ragger $\mathrm{K}$ et al (2016) White matter integrity in polydrug users in relation to attachment and personality: a controlled diffusion tensor imaging study. Brain Imaging Behav 10:1096-1107

29. Unterrainer $\mathrm{H}-\mathrm{F}$, Hiebler-Ragger $\mathrm{M}$, Koschutnig $\mathrm{K}$ et al (2017) Addiction as an Attachment Disorder: White Matter Impairment Is Linked to Increased Negative Affective States in Poly-Drug Use. Front Hum Neurosci 11:208

30. Wedekind D, Bandelow B, Heitmann S et al (2013) Attachment style, anxiety coping, and personalitystyles in withdrawn alcohol addicted inpatients. Subst Abuse Treat Prev Policy 8:1

31. Wyrzykowska E, Głogowska K, Mickiewicz K (2014) Attachment relationships among alcohol dependent persons. Alcohol Drug Addict 27:145-161

32. Zellner MR, Watt DF, Solms Met al (2011) Affective neuroscientific and neuropsychoanalytic approaches to two intractable psychiatric problems: Why depression feels so bad and what addicts really want. Neurosci Biobehav Rev 35:2000-2008

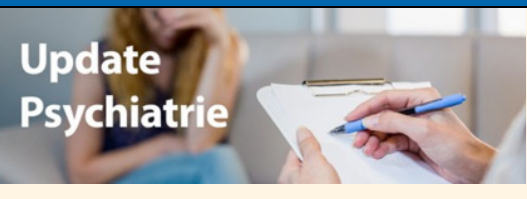

\section{Schon bestellt?}

Die Update Newsletter von SpringerMedizin.de zu Ihrem Fachgebiet liefern Ihnen regelmäßig Aktuelles und Wissenswertes speziell zu Ihrem Fachgebiet:

- CME-Beiträge aus den

Fachzeitschriften von Springer Medizin

- umfassende Übersichtsbeiträge und interessante Kasuistiken

- aktuelle internationale Studien

- Kongress-Highlights und ThemenSpecials

- News aus Berufs- und Gesundheitspolitik

Jetzt Newsletter kostenlos bestellen unter www.springermedizin.de/mynewsletters 